\title{
Field acoustic measurements of high-speed train sound along BTIR $^{\dagger}$
}

\author{
YU HuaHua* \& LI JiaChun* \\ Institute of Mechanics, Chinese Academy of Sciences, Beijing 100190, China
}

Received September 26 2012; accepted October 23, 2012; published online January 21, 2013

\begin{abstract}
In this paper, single-point field measurements of noise radiated from high-speed trains were performed at two sites along Beijing-Tianjin intercity railway (BTIR), aiming at acquiring the realistic acoustic data for validation and verification of physical model and computational prediction. The measurements showed that A-weighted sound pressure levels (SPLs) were between 80 and $87 \mathrm{dBA}$ as trains passed. The maximum noise occurred at the moment when the pantograph arrived, suggesting that pantograph noise was one of the most significant sources. Sound radiated from high-speed trains of BTIR was a typical broadband spectrum with most acoustic power restricted in the range of medium-high frequency from about $400 \mathrm{~Hz}$ to $5 \mathrm{kHz}$. Aerodynamic noise was shown to be the dominant one over other acoustic sources for high-speed trains.
\end{abstract}

field measurement, noise level, Beijing-Tianjin intercity railway, high-speed trains

PACS number(s): 43.28.Ra, 43.58.+z, 43.50.Rq, 47.40.Dc, 47.85.Lf

Citation: Yu H H, Li J C. Field acoustic measurements of high-speed train sound along BTIR. Sci China-Phys Mech Astron, 2013, 56: 474-482, doi: 10.1007/ s11433-013-5007-7

\section{Introduction}

In recent years, a number of high-speed railway lines operating at speeds of more than $300 \mathrm{~km} / \mathrm{h}$ have been rapidly developed in China, such as Beijing-Shanghai, WuhanGuangzhou and Zhengzhou-Xi'an express railway [1]. High-speed trains are evidently superior to conventional ones in travelers' speed and comfort during any type of journey. Hence, it can be anticipated that such a growth trends of infrastructure in high-speed railway will be maintained in China in the foreseeable future. However, noise generated by train and train-rail interactions may transmit into the carriage and thus reduce the comfort of passengers. When it radiates outward to ambient environments, surrounding residents alongside the railway will also be affected. For this reason, sound emission from high-speed

\footnotetext{
*Correspongding author (YU HuaHua, email: yuhuahua@imech.ac.cn; LI JiaChun, email: jcli05@imech.ac.cn)
}

$\uparrow$ Contributed by LI JiaChun (CAS Academician) trains has received increasing public scrutiny [2].

In general, high-speed trains consist of three kinds of acoustic sources [3], namely, traction motor system, rolling wheels and aerodynamic factors. They play different roles at different ranges of train speed. Relevant researches [4] have shown that noise due to traction motor system and rolling wheels vary proportionally to the first and third power of train speed, respectively. However, aerodynamic noise grows at the sixth power of train speed. Consequently, with the continuous increase of train speed, the contribution of aerodynamic noise will eventually exceed that of traction motor system noise and rolling noise and will finally become the dominant factor. Therefore, computational aeroacoustics (CAA) has been developed as an effective tool in the study of high-speed train noise. Concurrently, experimental measurements cannot be neglected in model validation and computation verification.

A series of external noise measurements were carried out on board a high-speed train by Bracciali et al. [5] using a specially designed device with microphones in the axlebox 
of a carriage in proximity to a single wheel. Barsikow [6] gave the principal results from noise measurements made with microphone arrays of different configurations and arrangements to find out acoustic sources on the Deutsche Bahn AG trains at the speed of from $60 \mathrm{~km} / \mathrm{h}$ to $280 \mathrm{~km} / \mathrm{h}$. Field measurements were performed by Kikuchi et al. [7] at two sites, one near a tunnel portal and the other in a fully open section, showing that major components of the low-frequency noise from the tunnel portal are impulsive micro-pressure waves and continuous pressure waves, while those in the open section are near-field aerodynamic pressure variations and far-field acoustic pressure waves. Field acoustic measurements of noise radiated by passing trains nearby viaduct railway were compared with various numerical noise predictions by Lui et al. [8]. Kitagawa et al. [9] attempted to validate TWINS model for rail and wheel noise prediction based on comparison with measurements for four different wheel/rail combinations. A field experiment was conducted by De Coensel et al. [10] to study the possible differences between conventional and high-speed trains in perceived noise annoyance. Wakabayashi et al. [11] performed acoustic measurement of Shinkansen high-speed test train (FASTECH360S, Z) to test countermeasures for the reduction of noise of high-speed train such as using low-noise pantograph, sound absorbing panels and pantograph noise insulation plates.

Only a few of previous studies in acoustic wind tunnel tests have been reported. Nagakura [12] performed wind tunnel sound tests by using an acoustic mirror and a $1 / 5^{\text {th }}$ model scale Shinkansen train to study the distribution of aerodynamic noise sources of a train. By installing microphone array in the shear layer of the train model, Yamazaki et al. [13] found that one of the primary noise sources was from the gap between carriages. Mizushima et al. [14] measured aerodynamic noise of broadband downstream the gap due to eddy separation and collision, further demonstrating that the noise reduction can be reached by rounding the downstream edge.

Beijing-Tianjin intercity railway (BTIR) is China's first high-speed railway operating at $350 \mathrm{~km} / \mathrm{h}$. The completion of this line as long as $120 \mathrm{~km}$ connecting two metropolitans considerably facilitates residents' travelling time. About $87 \%$ sections of the railway are built on viaducts while the rest are based on subgrades. The main type of trains running on BTIR is CRH3 as shown in Figure 1(a). It contains 8 coaches of $3.3 \mathrm{~m}$ in width, $3.89 \mathrm{~m}$ in height and $25 \mathrm{~m}$ in length for each carriage. From the diagram we can find that the train head shape, designed as a streamlined body of smooth bullet type. Figure 1(b) exhibits quantitative variation of the cross-section areas of train head. It is believed that a linear variation of train head cross-section areas possesses excellent aerodynamic performance with remarkably reduced drag and noise. Two pantographs are installed on the roof of the second coach from both ends, but only one of them at the rear is uplifted in operation. Noise radiated from high-speed trains of BTIR mainly consists of four parts, that is, wheel-rail system, current-collection system, aerodynamic noise and vibration of viaduct bridges [15-17]. Current-collection system noise involves sliding noise of pantograph on electrified wires, electric arc noise and aerodynamic noise of pantograph system.

Previous field measurements were seldom performed for high-speed trains at the speed of more than $300 \mathrm{~km} / \mathrm{h}$, while wind tunnel tests usually were conducted at lower Reynolds number. It seems that field acoustic measurements of high-speed trains are necessary in the verification of CAA models and mechanism analysis. In this paper, field measurements of noise radiated from high-speed trains were carried out at two sites of BTIR nearby Yizhuang and Yongledian stations, respectively. A handheld sound level meter was used in the acoustic measurements. Six groups of data such as time histories of SPLs and A-weighted SPLs were obtained during the pass-by of $\mathrm{CRH} 3$ type trains at the speed of more than $300 \mathrm{~km} / \mathrm{h}$. One-third octave band frequency spectra of noise are also analyzed.

\section{Field acoustic measurements by BTIR}

\subsection{Instruments for acoustic measurements}

A handheld Casella CEL-490 type real-time sound level meter (SLM) as shown in Figure 2 was used in the single-
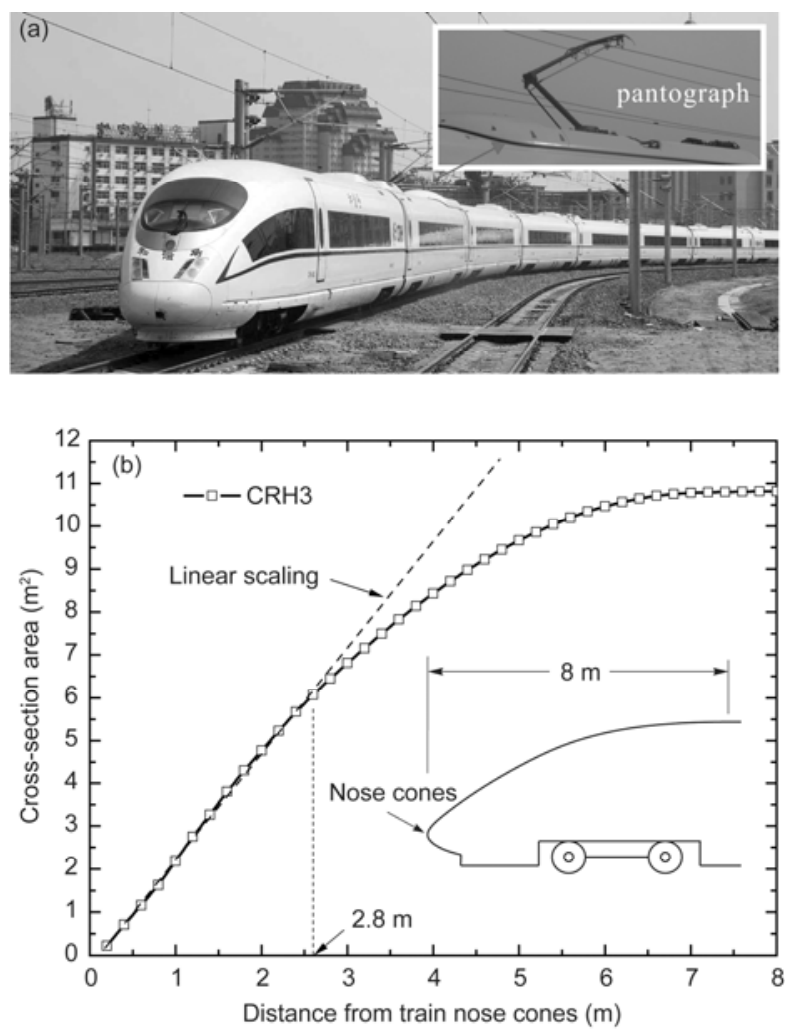

Figure 1 Details of CRH3 type high-speed train. (a) Outline; (b) crosssection areas variations of train head. 


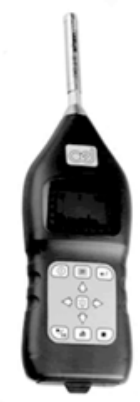

Figure 2 Handheld Casella CEL-490 real-time sound level meter (SLM).

point noise measurements. The lower part of the instruments is an operation panel with LCD backlit screen to displays the current test conditions and results. A $1 / 2$ inch or $1 / 4$ inch electret microphone is installed on the top of the instruments that receives sound signals and transform them into electrical signals.

The major technical specifications of Casella CEL-490 real-time sound level meter are listed in Table 1. The SLM has a resolution of $0.1 \mathrm{~dB}$ with the maximum measurement range of $140 \mathrm{~dB}$. DSP sampling rate is $67.2 \mathrm{kHz}$ which ensures that the maximum frequency response can be as high as $30 \mathrm{kHz}$. The smallest sampling interval is $10 \mathrm{~ms}$ which means 100 samples of data will be obtained in just one second. This is significant in our measurements because the time is only about $2 \mathrm{~s}$ for passing-by trains at the speed of more than $300 \mathrm{~km} / \mathrm{h}$.

In order to avoid the influence of wind in measurement processes, a wind cap made of porous sponge was used to cover the electret microphone. The wind cap can prevent the occurrence of turbulence flow by the wind and is capable of eliminating the effect of wind noise. However, the wind cap may lead to some acoustic attenuation of sound pressure levels (SPLs) at the same time. The attenuation magnitude and frequency characteristics can be determined by comparing the results between the cases with and without wind cap in windless air. The acoustic attenuation magnitude of wind cap is small as compared to SPLs of normal conversation by verification. Therefore, the attenuation effect is ignored in our field measurements.

To obtain the most accurate results, the instruments were be calibrated initially for the response of a known directional microphone, which is called calibration check. Both before and after a measurement, a calibration check of the instruments were be made using a CEL-110 or CEL-111 calibrator for SLMs with first level accuracy and a CEL-110 or CEL-112C calibrator for SLMs with second level accuracy. All of the calibrators supplied a calibration level of $114 \mathrm{~dB}$, while the CEL-110 or CEL-111 calibrator provided a nominal level of $94 \mathrm{~dB}$ at the same time. However, the accurate value for calibration of the instruments depended directly on the microphone type and atmospheric conditions in practical applications.

\subsection{Field measurements details}

The measuring procedures were carried out at two different sites along BTIR, which were nearby Yizhuang station and Yongledian station, respectively. Figure 3 shows the schematic diagram of acoustic measurements at these two sites, in which Figure 3(a) denotes Yizhuang station and Figure $3(\mathrm{~b})$ is Yongledian station.

Table 1 Key technical specifications of Casella CEL-490 real-time SLM

\begin{tabular}{|c|c|}
\hline Parameters & Value \\
\hline \multirow{3}{*}{ Acoustic accuracy (Aplicable standards) } & ANSI S1.4:R1997, ANSI S1.43:1996 \\
\hline & IEC 60651:1994, IEC 60804:2000 \\
\hline & IEC 61672:2002, IEC 61260:Class 0 \\
\hline Measurement mode & Broadband, octave band, 1/3 octave band \\
\hline Measurement range & 0-140 dB RMS, $143.3 \mathrm{~dB}$ peak \\
\hline Resolution & $0.1 \mathrm{~dB}$ \\
\hline \multirow{2}{*}{$\begin{array}{l}\text { Broadband mode measured parameters } \\
\text { ( } x=\text { frequency weighting, } y=\text { time weighting) }\end{array}$} & $L_{\mathrm{Ceq}}-L_{\mathrm{Aeq}}, L_{\mathrm{EPd}}, \mathrm{TWA}$ \\
\hline & $5 \times L_{\mathrm{N}} \%($ user selectable $(0.1-99.9) \%)$ \\
\hline \multirow{2}{*}{$\begin{array}{l}\text { Octave and } 1 / 3 \text { octave band mode measured parameters } \\
(x=\text { frequency weighting, } y=\text { time weighting) }\end{array}$} & $L_{x y}, L_{\text {Xeq }}, L_{x y \max }, L_{x y \min }, L_{x \mathrm{pk}}$ \\
\hline & $5 \times L_{\mathrm{N}} \%($ user selectable $(0.1-99.9) \%)$ \\
\hline \multirow{2}{*}{ Microphone details } & $1 / 2$ inch electret microphone \\
\hline & $1 / 4$ inch electret microphone \\
\hline Frequency response & $6 \mathrm{~Hz}-30 \mathrm{kHz}( \pm 3 \mathrm{~dB})$ \\
\hline Time weightings & Fast, Slow and Impulsive \\
\hline Weightings $(Q)$ & $Q=3,7,8$ or 9 \\
\hline DSP sampling rate & $67.2 \mathrm{kHz}$ \\
\hline \multirow{3}{*}{ Sampling intervals (Time history profiles) } & $10,20,50,100,250,500 \mathrm{~ms}$ \\
\hline & $1,5,10,15,20,30 \mathrm{~s}$ \\
\hline & $1,5,10,15,20,30 \mathrm{~min}$ \\
\hline
\end{tabular}


(a)

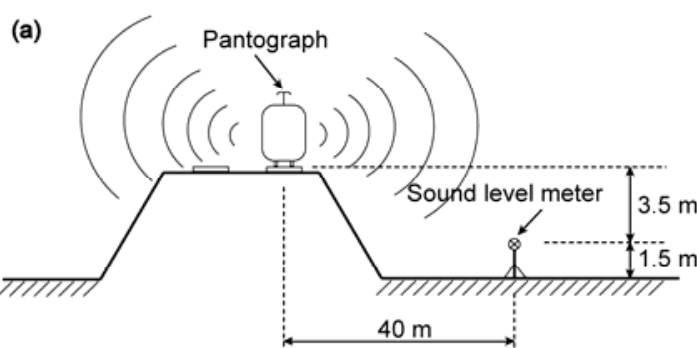

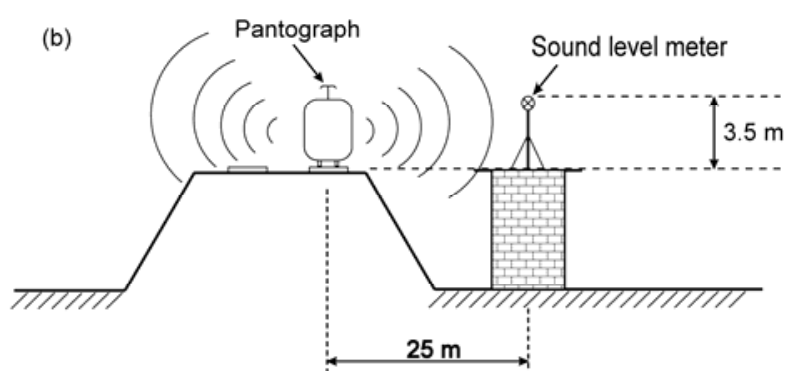

Figure 3 Schematic diagram of noise measurements from high-speed trains at two different sites along BTIR. (a) Yizhuang station; (b) Yongledian station.

At the location nearby Yizhuang station, the rail track is constructed on subgrades, about $5 \mathrm{~m}$ higher than the surrounding ground level. Restricted by geological conditions, the measurements were not located at the standard position. The sound level meter was installed beside the railway at a place $1.5 \mathrm{~m}$ above the ground (or $3.5 \mathrm{~m}$ below the surface of rail track) and $40 \mathrm{~m}$ from the centerline of the rail track. However, there was a single-storey building in close proximity to the subgrades nearby Yongledian station. Then, the sound level meter was installed on the roof of the building about $25 \mathrm{~m}$ from the centerline of the rail track and $3.5 \mathrm{~m}$ above the rail track. Figure 4 shows views of field measurements at nearby Yongledian station.

There were no barriers on both sides of the railway at these two sites. Sound directly radiated to the measurement points without any acoustic shield or barriers leading to attenuation or reflection of sonic wave. The measurement points were set in the completely open semi-infinite space. In the present measurements, time weighting was set to be fast mode while the sampling interval was chosen as $10 \mathrm{~ms}$, which means 100 samples will be obtained in just one second.
The magnitude of acoustic level is presented in terms of sound pressure level in the decibel scale $(\mathrm{dB})$, defined as:

$$
S P L=20 \lg \frac{p_{\mathrm{e}}}{p_{\text {ref }}}
$$

where $p_{\mathrm{e}}$ is the effective sound pressure corresponding to root-mean squared sound pressure; $p_{\text {ref }}$ denotes a reference pressure which usually takes the value of threshold of human audibility in air, $p_{\text {ref }}=2 \times 10^{-5} \mathrm{pa}=20 \mu \mathrm{pa}$. However, the sensation of the human ear to loudness of sound is strongly dependent on frequency as well as its magnitude. More specifically, the human ear response is more sensitive to sounds in the frequency range between $1 \mathrm{kHz}$ and $4 \mathrm{kHz}$ than those outside of this range. Consequently, sound level meter was often fitted with a filter (i.e., frequency weighting) network so that the ultimate response to frequency resemble more what the human hear is capable of. This filtering network working in the same way as the human ear does attenuates low frequency and high frequency components and leaves middle frequencies almost unchanged. If A-weighting filter is used (see Figure 5), SPL will be alternatively
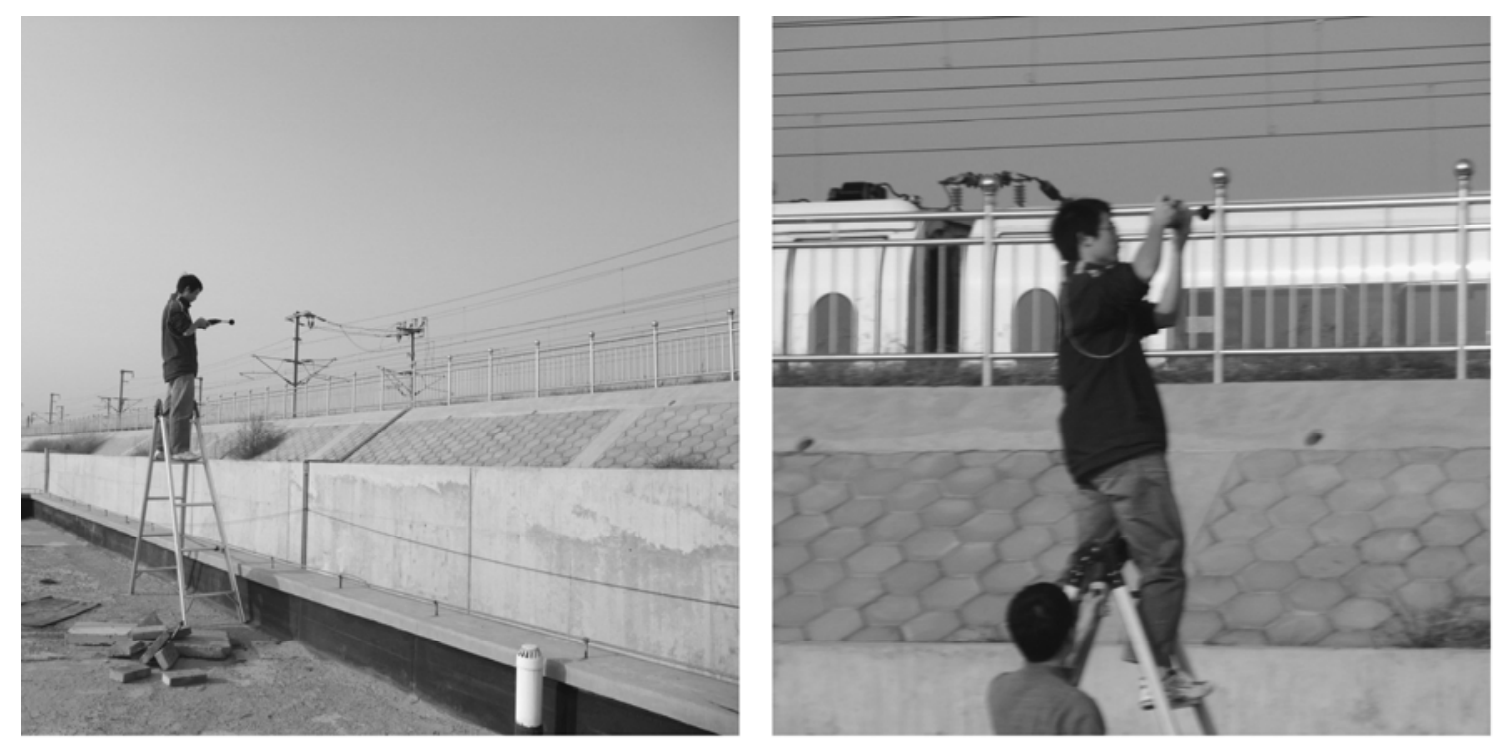

Figure 4 Views of field measurements at the location nearby Yongledian station. 


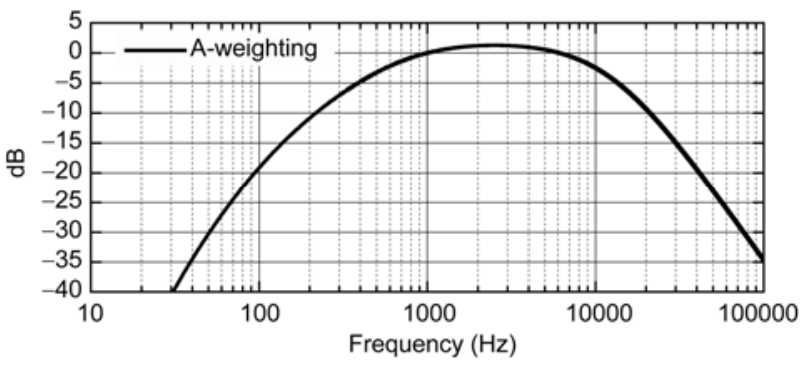

Figure 5 A-weighting filter response curve.

given in terms of $\mathrm{dBA}$ that we call A-weighted SPLs. A-weighted SPLs, widely used in acoustic measurements, can well reflect the actual sensation of noise loudness for the human ear. In frequency analysis of acoustic signals, audible range from $20 \mathrm{~Hz}$ to $20 \mathrm{kHz}$ is usually separated into a few frequency bands. If the upper limit is twice that of the lower limit, we term this the octave band which is further separated into three ranges, referred to as one-third octave bands. In the present measurements one-third octave band frequency mode was chosen for frequency spectrum analysis.

\section{Results and discussion}

\subsection{Results at Yizhuang station}

Three measurements were performed nearby Yizhuang station at the same location. Trains were all CRH3 type high-speed trains running from Beijing to Tianjin at the speed of $330 \mathrm{~km} / \mathrm{h}$. Time histories of measured SPLs during pass-by of high-speed train are shown in Figure 6, in which three lines represent three different times of measurements, respectively. Measurements began at 1-3 s before the train head arrived and stopped at the moment after the train tail passed at about 3 seconds. The time interval of train passage was about 2.2 seconds from $3.2 \mathrm{~s}$ to $5.4 \mathrm{~s}$ as shown in Figure 6 . The variation trends of three noise profiles are similar. SPLs increased rapidly from about 80 to $95 \mathrm{~dB}$ as the train head approached the measurement point, then fluctuated at a flat level around $97 \mathrm{~dB}$ during the period of train passage, and dropped down to $80 \mathrm{~dB}$ gradually after train tail passed.

Figure 7 shows time histories of A-weighted SPLs corresponding to Figure 6. The magnitudes of A-weighted SPLs during the passage of trains are between 80 and 85 dBA. The maximum value of A-weighted SPLs corresponded to the position of pantograph, suggesting that pantograph was one of the most critical noise sources in these cases. Pantograph noise involves sliding noise of pantograph on electrified wires, electric arc noise and aerodynamic noise of pantograph system. Pantograph is mainly composed of structural slender components (such as rod-shaped members) which disturb the flow field and form complicated unsteady wake flow. The periodic vortex shed-

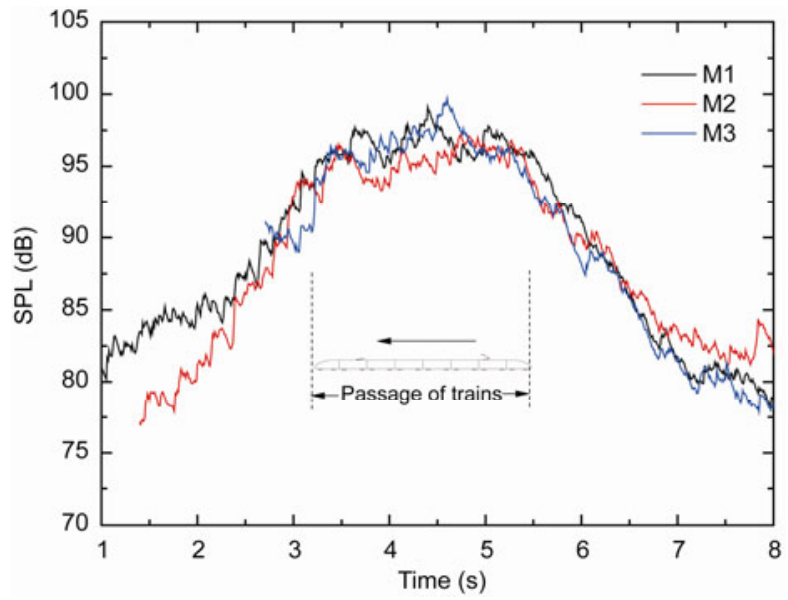

Figure 6 Time histories of SPLs measured with Casella CEL-490 real time SLM at a distance of $40 \mathrm{~m}$ to the centerline of the track during a pass-by of a CRH3 high-speed train at $330 \mathrm{~km} / \mathrm{h}$, three lines represent three different times of measurements, respectively.

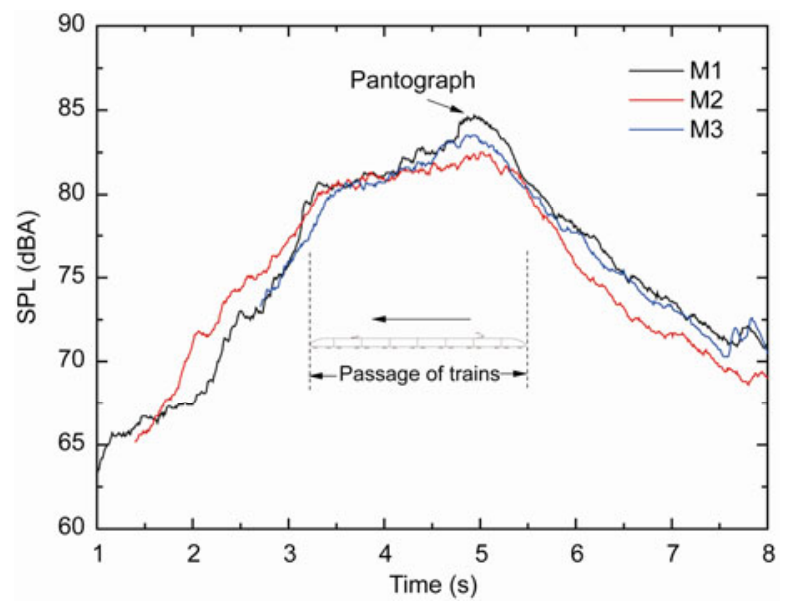

Figure 7 Time histories of A-weighted SPLs measured with Casella CEL-490 real time SLM at a distance of $40 \mathrm{~m}$ to the centerline of the track during a pass-by of a CRH3 high-speed train at $330 \mathrm{~km} / \mathrm{h}$, three lines represent three different times of measurements, respectively.

ding from slender components and the complicated interactions between them are the main mechanisms for this aerodynamic noise. The reduction of aerodynamic noise radiated by pantograph is the most critical factor in a total noise reduction program.

Typical one-third octave frequency spectra for three measurements are shown in Figure 8. The frequency spectra correspond to the time of around $5 \mathrm{~s}$ in Figure 7 where the maximum A-weighted noise level occurred. From Figure 8, we can determine that noise radiated from high-speed trains of BTIR was a typical broadband noise. However, there were two peaks in frequency spectra, one at $100 \mathrm{~Hz}$ and the other at approximately $1 \mathrm{kHz}$. Low frequency components $(100 \mathrm{~Hz})$ mainly come from wheel-rail noise while mediumhigh frequencies $(1 \mathrm{kHz})$ are dominated by aerodynamic noise. SPL at $1 \mathrm{kHz}$ was $5-10 \mathrm{~dB}$ higher than that of $100 \mathrm{~Hz}$. 


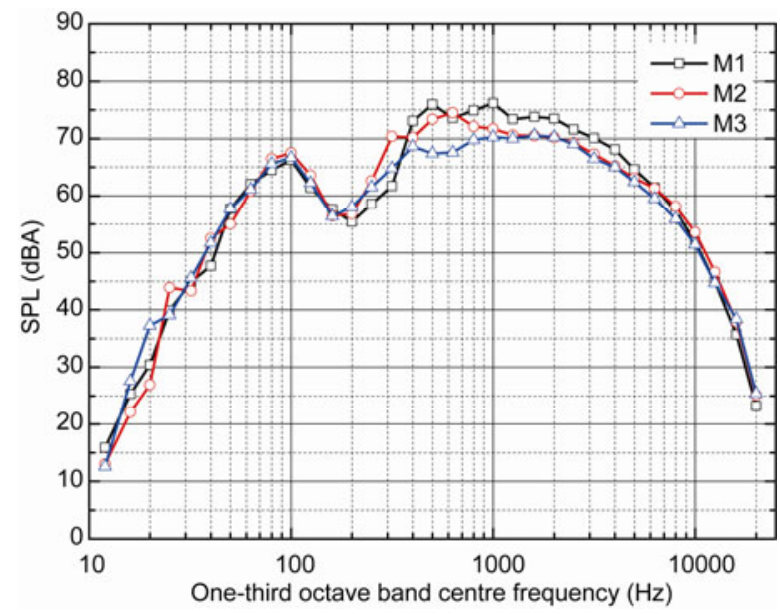

Figure 8 Typical 1/3 octave frequency spectra of noise radiated by high-speed train of BTIR at Yizhuang station for three measurements.

There was a seeming drop at $200 \mathrm{~Hz}$ for all three measurements. Very probably it was partially due to the influence of environment as the measurements point was taken below the subgrade and it may shield sound waves of some particular frequency. Most of the acoustic energy was concentrated in the medium-high frequency ranging from about $400 \mathrm{~Hz}$ to $5 \mathrm{kHz}$. This phenomenon is primarily attributed to the fact that aerodynamic noise contributes majority of total amount as train speed exceeds $300 \mathrm{~km} / \mathrm{h}$.

\subsection{Results at Yongledian station}

Another three measurements were performed nearby Yongledian station at the same location. Trains were all CRH3 type high-speed trains running from Tianjin to Beijing at the speed of $350 \mathrm{~km} / \mathrm{h}$. Time histories of measured SPLs during pass-by of high-speed trains are shown in Figure 9 , in which three lines represent three different measurements, respectively. The results are all linear (nonweighted) in frequency. The time interval of train passage was about $2 \mathrm{~s}$ from $1.5 \mathrm{~s}$ to $3.5 \mathrm{~s}$ as shown in Figure 9. The variation trends of three noise profiles were similar. SPLs increased rapidly from about 80 to $95 \mathrm{~dB}$ as train head approached the measurement point, then fluctuated at a flat level around $95 \mathrm{~dB}$ during the period of train passage, and dropped down to $80 \mathrm{~dB}$ gradually after train tail passed.

Figure 10 shows time histories of A-weighted SPLs corresponding to Figure 9. The A-weighted SPLs during the passage of trains were between 82 and $87 \mathrm{dBA}$. It was about 2 dBA more than that of YiZhuang station because the speed was higher and the measurement location was nearer to the train. The profiles of M1 and M2 were similar to that of Figure 7 while M3 was slightly altered from the previous two. The noise profile of M3 appeared as the shape of a double camel peak. The two peak values corresponded to

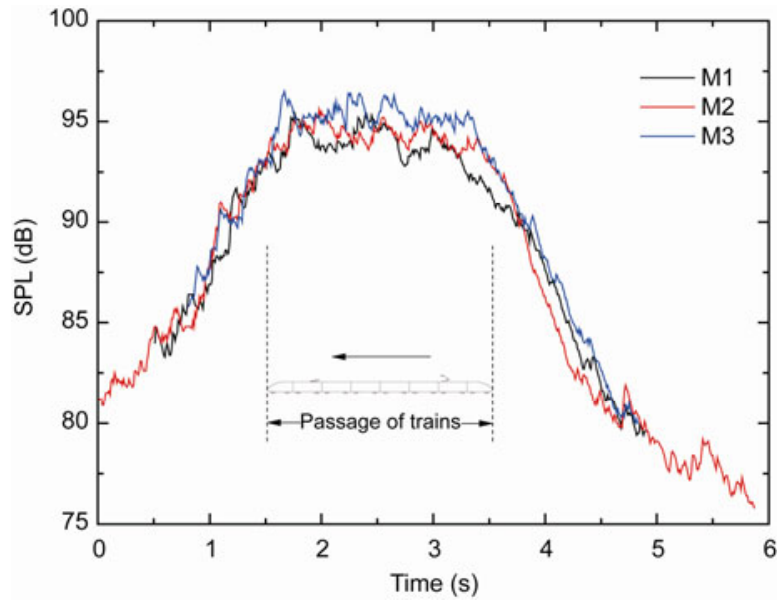

Figure 9 Time histories of SPLs measured with Casella CEL-490 real time SLM at a distance of $25 \mathrm{~m}$ to the centerline of the track during a pass-by of a CRH3 high-speed train at $350 \mathrm{~km} / \mathrm{h}$, three lines represent three different times of measurements, respectively.

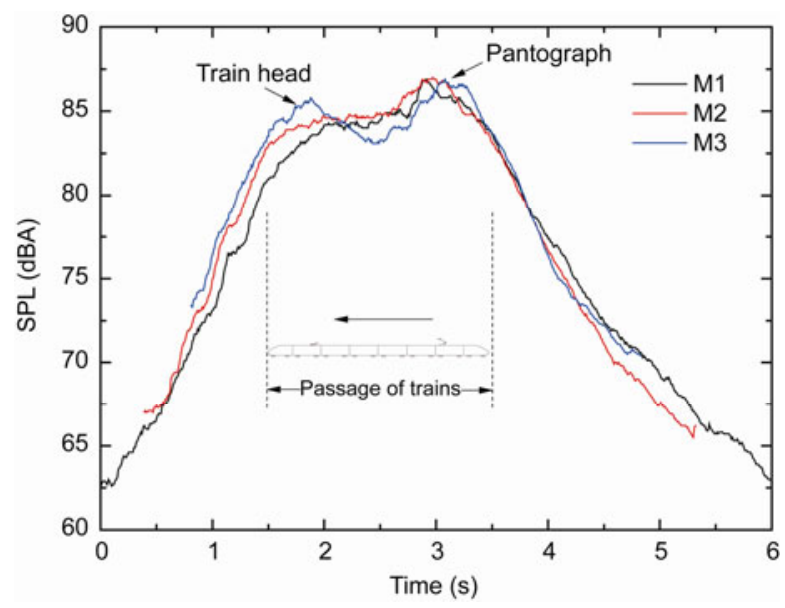

Figure 10 Time histories of A-weighted SPLs measured with Casella CEL-490 real time SLM at a distance of $25 \mathrm{~m}$ to the centerline of the track during a pass-by of a CRH3 high-speed train at $350 \mathrm{~km} / \mathrm{h}$, three lines represent three different times of measurements, respectively.

the position of train head and pantograph, respectively. The maximum value of A-weighted SPLs also occurred corresponding to the position of pantograph.

Typical one-third octave frequency spectra for three measurements are shown in Figure 11. The frequency spectra correspond to the time of around $3 \mathrm{~s}$ in Figure 10 where the maximum A-weighted noise level occurred. The noise radiated from high-speed trains was a typical broadband noise. There were no obvious peaks in frequency spectra. SPLs increased gradually from low frequency to around 1 $\mathrm{kHz}$ and decreased rapidly after $5 \mathrm{kHz}$. Similar to the results at Yizhuang station, most of the acoustic energy was concentrated in the medium-high frequency ranging from about $400 \mathrm{~Hz}-5 \mathrm{kHz}$. Aerodynamic noise was the dominant one among various sources for high-speed trains. 


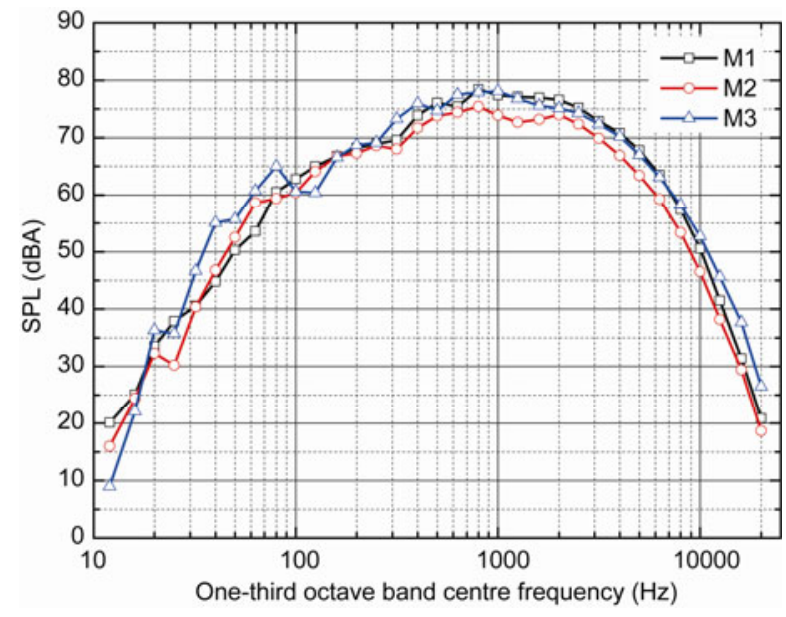

Figure 11 (Color online) Typical one-third octave band frequency spectra of noise radiated by high-speed train of BTIR at Yongledian station for three measurements.

\subsection{Discussion}

A-weighted SPLs during the pass-by of train were $10 \mathrm{dBA}$ higher than that at the moments when train approached and left. What mechanism enables for this type of difference? Figures 12(a) and (b) show 1/3 octave band frequency spectra of noise measured at Yongledian station for three different moments of M1 and M3, respectively. Line with solid squares (denoted by B) is the result at the time point of about $3 \mathrm{~s}$ in Figure 10, corresponding to the maximum value of A-weighted SPLs. The other two lines with solid circles and triangles (denoted by $\mathrm{A}$ and $\mathrm{C}$ ) represent data at around 1 and $4 \mathrm{~s}$ in Figure 10, corresponding to the moments when train approached and left, respectively. Overall SPLs at these two moments were all approximately $75 \mathrm{dBA}$. From these two figures, one can readily determine the difference of frequency spectra between them. The frequency spectra results of $\mathrm{A}$ and $\mathrm{C}$ were consistent. The magnitude at low frequency region (lower than $200 \mathrm{~Hz}$ ) of B was higher than that of $\mathrm{A}$ and $\mathrm{C}$ by the magnitude of around $5 \mathrm{dBA}$. However, in medium-high frequency region (higher than $400 \mathrm{~Hz}$ ) the difference was more than $10 \mathrm{dBA}$. Thus, the magnitude of acoustic energy increased more in medium-high frequency region than in low frequency region when train arrived. It was implied in the previous sections that medium-high frequency components were primarily from aerodynamic noise. Therefore, we can arrive at the conclusion that aerodynamic noise contributes most to the increase of noise level during the pass-by of high-speed trains.

Aerodynamic noise contributes majority of total amount of sound at the speed of more than $300 \mathrm{~km} / \mathrm{h}$. By averaging A-weighted SPLs of three measurements conducted at each place, we can obtain two lines in Figure 13 representing profiles of averaged A-weighted SPLs measured at two locations, respectively, where the solid and dashed lines denote the measurements of nearby Yongledian and Yizhuang
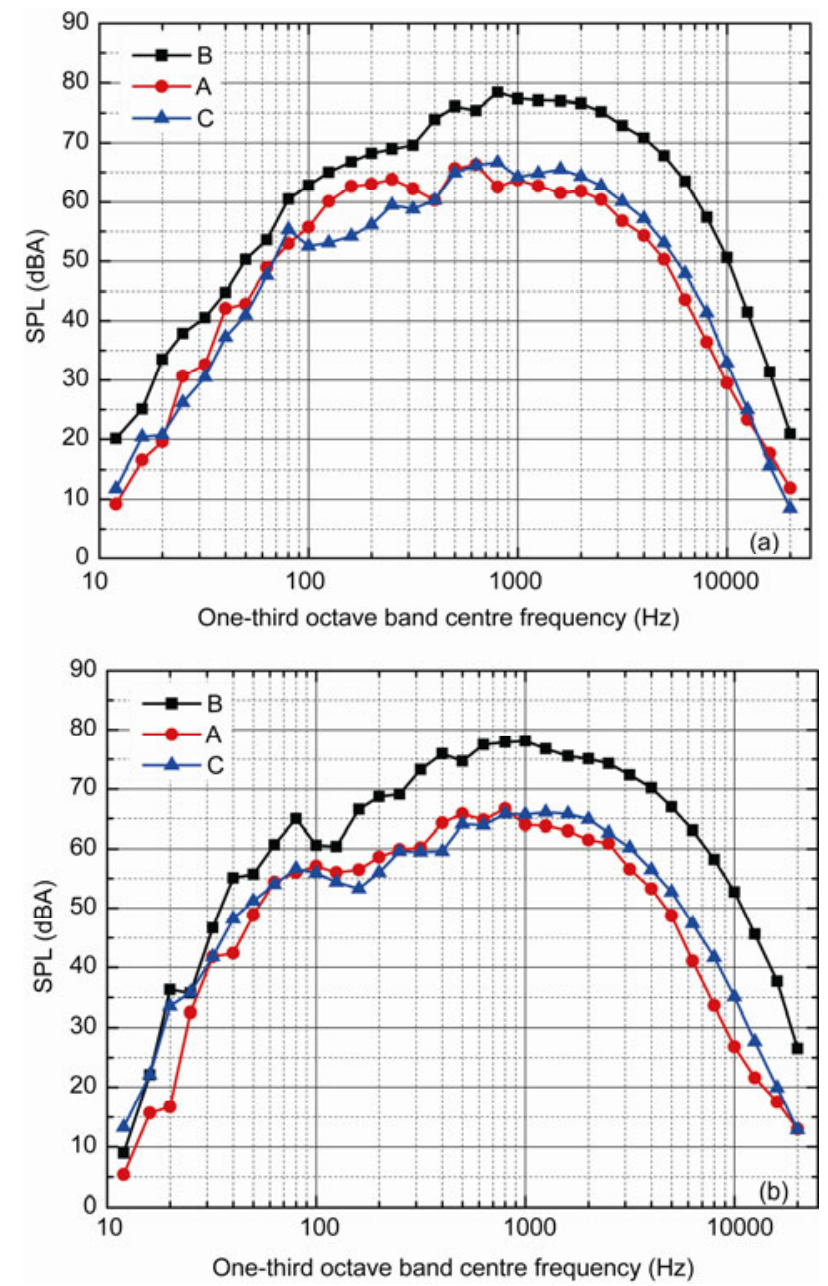

Figure 12 (Color online) 1/3 octave band frequency spectra of noise radiated by high-speed train of BTIR for three moments at Yongledian station (a) M1; (b) M3.

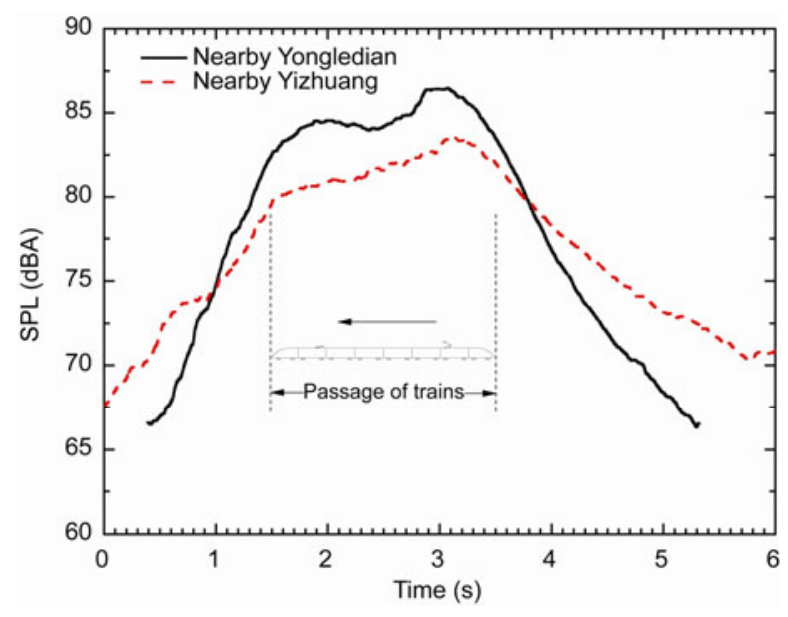

Figure 13 (Color online) Profiles of averaged A-weighted SPLs measured at two locations.

stations. The value of difference between two lines during the passage of trains (from $1.5 \mathrm{~s}$ to $3.5 \mathrm{~s}$ ) was between 1.5 and $4 \mathrm{dBA}$ as shown in Figure 14 with the mean value of 


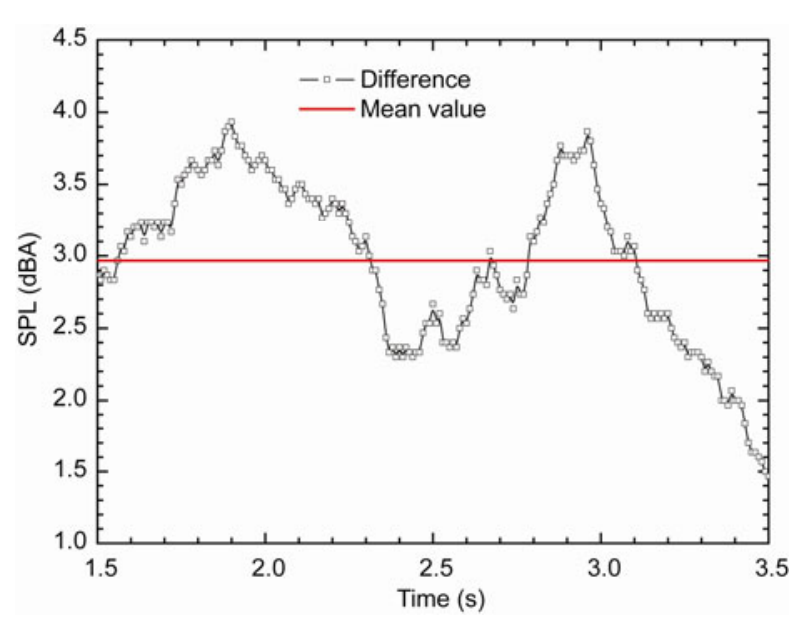

Figure 14 (Color online) Difference between averaged A-weighted SPLs measured at two locations.

difference during this period being $2.97 \mathrm{dBA}$.

According to relevant researches of former investigators, aerodynamic noise increases proportional to the $6^{\text {th }}$ power of train speed [4], that is

$$
p_{\mathrm{e}} \propto U^{6}
$$

thus we have

$$
\frac{p_{1 \mathrm{e}}}{p_{2 \mathrm{e}}}=\left(\frac{U_{1}}{U_{2}}\right)^{6},
$$

where $p_{1 \mathrm{e}}$ and $p_{2 \mathrm{e}}$ denote the effective sound pressure at two different speeds $U_{1}$ and $U_{2}$, respectively.

The difference of SPLs between two velocities then satisfies the following expression;

$$
S P L_{1}-S P L_{2}=20 \lg \frac{p_{1 \mathrm{e}}}{p_{2 \mathrm{e}}}=120 \lg \frac{U_{1}}{U_{2}} .
$$

Therefore, by substituting $U_{1}=350 \mathrm{~km} / \mathrm{h}$ and $U_{2}=330 \mathrm{~km} / \mathrm{h}$ into the above equation, we can acquire the difference of SPLs between speeds at $350 \mathrm{~km} / \mathrm{h}$ and $330 \mathrm{~km} / \mathrm{h}$ as 3.07 $\mathrm{dBA}$, which is responsible for the mechanism of difference in the previous measurements for different speed of trains. Field measurements further imply that the sixth power law of sound pressure is valid and can be used to predict SPL of trains at different speeds.

\section{Application in the verification of numerical simulations}

The authors have conducted a series of numerical simulations of aerodynamic noise radiated by high-speed trains. As a paradigm, the acoustic data in the previous field measurements are applied to verify the reliability of computational results.
Due to the limitations of computation resources, a simplified train model with three coaches was used in the computation with the geometry of train head and carriage kept unchanged with CRH3. However, the pantograph in this case was located on the roof of the second coach. The computational domain was discretinized by a hybrid grid blended with unstructured tetrahedrons and triangular prisms. The total number of grid cells was about 12 million. Aerodynamic noise at the speed of $350 \mathrm{~km} / \mathrm{h}$ was calculated by using hybrid NLAS/FW-H [18] method.

The comparisons of A-weighted SPLs between computations and measurements are shown in Figure 15, in which the solid lines are three measurement results in the previous section while the red line with hollow circles is computational results for trains of three coaches. The maximum SPL corresponds to the moment when pantograph arrives. We can see that the computational profile only agrees well with measurements after $2.75 \mathrm{~s}$. Thus, this kind of difference between them comes from the length of train with different number of coaches. By translating SPLs between 2.25 and $2.75 \mathrm{~s}$ to 1 and $1.5 \mathrm{~s}$ (corresponding to train head of computational model) and extending the curve to $2.75 \mathrm{~s}$ based on the measurements, we can reproduce a new line denoted by hollow squares. This profile can partially represents computational results of train with 8 coaches. In this way, we can find that the profile shows good agreement with measured results. Figure 16 exhibits the comparison of frequency spectra in good agreement between simulation and measurements. Based on such verifications, we can conclude that our numerical simulations can provide acceptable results in the predictions of aerodynamic noise radiated by high-speed trains.

\section{Conclusions}

Single point field acoustic measurements of noise radiated

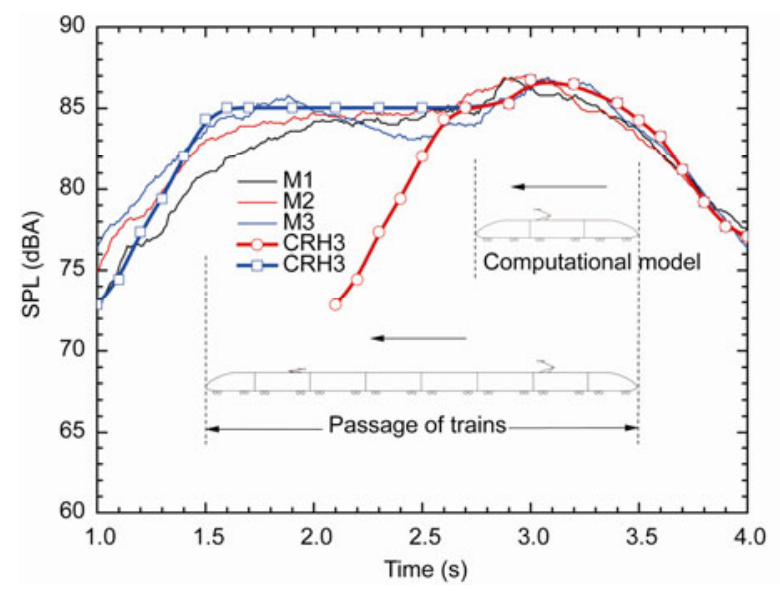

Figure 15 (Color online) Comparisons of A-weighted SPLs between numerical simulations and measurements. 


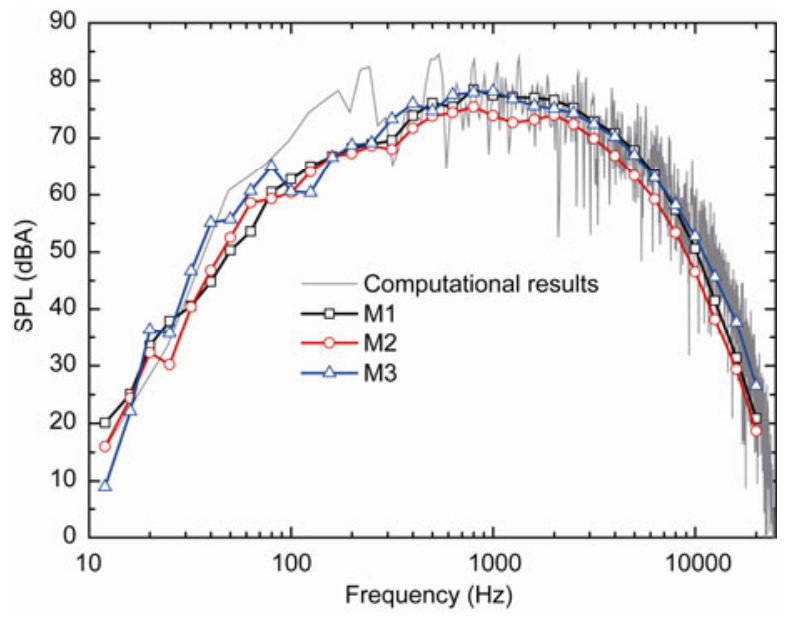

Figure 16 (Color online) Comparisons of frequency spectra between numerical simulations and measurements.

by pass-by high-speed trains were performed at two sites along BTIR using a handheld Casella CEL-490 type real-time sound level meter. In accordance with measured data analysis and mechanism analysis, the following conclusions can be drawn.

Firstly, the variation trends of noise profiles were similar for the six measurements at two locations. SPLs quickly increased from 80 to $95 \mathrm{~dB}$ as train head approached, then fluctuated at a flat level around $95 \mathrm{~dB}$ during the period of train passage, and gradually dropped down to $80 \mathrm{~dB}$ when train tail left.

Secondly, A-weighted SPLs were between 80 and 87 $\mathrm{dBA}$ during the passage of trains. The maximum noise occurred at the moment when the pantograph as one of the most significant aerodynamic acoustic source arrived. Therefore, countermeasures should be taken to reduce aerodynamic noise radiated by pantograph, which accounts for it becoming the most critical factor in a total noise reduction program.

Thirdly, noise radiated from high-speed trains of BTIR was a typical broadband one with most acoustic energy restricted in the range of medium-high frequency from about $400 \mathrm{~Hz}$ to $5 \mathrm{kHz}$. Aerodynamic noise was dominant over various other sources for high-speed trains.

Fourthly, the magnitude of acoustic energy in medium-high frequency region increased more rapid than in low frequency region when train arrived. Medium-high frequency components of SPLs primarily came from aerodynamic noise, which indicated that aerodynamic factors contributed most to the rise of SPL as speed increased during the passage of high-speed trains.

Lastly, the verification of numerical simulation on the basis of comparison with field acoustic measurements suggests that the CAA model is reliable and the computational accuracy is acceptable in the prediction of aerodynamic noise radiated by high-speed trains.
This work was supported by the National Key Technology R\&D Program (Grant No. 2009BAG12A03) and Knowledge Innovation Project of Chinese Academy of Sciences of China (Grant No. KJCX2-EW-L02-1). The authors appreciate CHEN QiSheng, YANG Yan, YANG QianSuo for their valuable assistance and cooperation in the field measurements.

1 Chen J G, Xia H, Cai C X, et al. Test and analysis of high-speed trains induced environmental noise and sound barriers (in Chinese). J Vib Eng, 2011, 24(3): 229-234

2 Gao J. Investigation of method for high-speed railway noise control (in Chinese). Railway Transport Economy, 2006, 28(7): 83-85

3 Talotte C, Gautier P E, Thompson D J, et al. Identification, modeling and reduction potential of railway noise sources: A critical survey. $\mathbf{J}$ Sound Vib, 2003, 267(2): 447-468

4 Ikeda M. The development of low noise pantograph (in Chinese). Foreign Locomotive Rolling Stock Tech, 1995, 5: 30-31

5 Bracciali A, Ciuffi L, Ciuffi R, et al. Continuous external train noise measurements through an on-board device. Proceedings of the Institution of Mechanical Engineers, Part F-J Rail Rapid Transit, 1994, 208(1): 23-31

6 Barsikow B. Experiences with various configurations of microphone arrays used to locate sound sources on railway trains operate by the DB AG. J Sound Vib, 1996, 193(1): 283-293

7 Kikuchi K, Iida M, Takasaki T, et al. Field measurement of wayside low-frequency noise emitted from tunnel portals and trains of high-speed railway. J Low Frequency Noise Vib Active Control, 2005, 24(4): 219-231

8 Lui W K, Li K M, Ng P L, et al. A comparative study of different numerical models for predicting train noise in high-rise cities. Appl Acoust, 2006, 67(5): 432-449

9 Kitagawa T, Thompson D J. Comparison of wheel/rail noise radiation on Japanese railways using the TWINS model and microphone array measurements. J Sound Vib, 2006, 293(3-5): 496-509

10 De Coensel B, Botteldooren D, Berglund B, et al. Experimental investigation of noise annoyance caused by high-speed trains. Acta Acust United Acust, 2007, 93(4): 589-601

11 Wakabayashi Y, Kurita T, Yamada H, et al. Noise Measurement Results of Shinkansen High-Speed Test Train (FASTECH360S, Z). In: Schulte-Werning B, Thompson D, Gautier P E, et al., eds. Noise and Vibration Mitigation for Rail Transportation Systems, Notes on Numerical Fluid Mechanics and Multidisciplinary Design. Munich, Germany: Proceedings of the 9th International Workshop on Railway Noise, 2007. 63-70

12 Nagakura K. Localization of aerodynamic noise sources of Shinkansen trains. J Sound Vib, 2006, 293(3-5): 547-556

13 Yamazaki N, Takaishi T, Toyooka M, et al. Wind Tunnel Tests on the Control of Aeroacoustic Noise from High Speed Train. Noise Measurement Results of Shinkansen High-Speed Test Train (FASTECH360S, Z). In: Schulte-Werning B, Thompson D, Gautier P E, et al., eds. Noise and Vibration Mitigation for Rail Transportation Systems, Notes on Numerical Fluid Mechanics and Multidisciplinary Design. Munich, Germany: Proceedings of the 9th International Workshop on Railway Noise, 2007. 33-39

14 Mizushima F, Kurita T, Kato C, Iida A. Reduction of aerodynamic noise from a train car gap. Noise Control Eng J, 2008, 56(6): 460464

15 Zhang S G. Noise mechanism, sound source localization and noise control of $350 \mathrm{~km} \cdot \mathrm{h}^{-1}$ high-speed train (in Chinese). China Railway Sci, 2009, 30(01): 86-90

16 Deng J, He Y L, Yang L Z, et al. Noise test and analysis for $350 \mathrm{~km} / \mathrm{h}$ high-speed train running on different types of railroad lines (in Chinese). Noise Vib Control, 2010, 30(6): 119-122

17 Dong H S, He Y L, Yang L Z, et al. Noise test and analysis of 350 $\mathrm{km} / \mathrm{h}$ high-speed train with different distances (in Chinese). Noise Vib Control, 2012, 32(1): 59-61

18 Paul B, Enrico R, Mauro C, et al. Towards a generalized non-linear acoustics solver. AIAA Paper, 2004, AIAA-2004-3001 